\title{
Malignant insulinoma in a patient with hypoglycemia
}

\author{
Tianqi Wang MD, Albert Vu MD, Laurie Mereu MD, Mahua Ghosh MBBS PhD
}

- Cite as: CMAJ 2022 March 7;194:E332-5. doi: 10.1503/cmaj.211002

A 67-year-old man presented to the emergency department with a 2-hour history of generalized weakness, nausea and confusion. The patient had no diaphoresis, palpitations or tremors, and felt well earlier in the day. He had a medical history of congestive heart failure with reduced ejection fraction, chronic kidney disease and pulmonary hypertension, and was taking apixaban, atorvastatin, furosemide, metoprolol and sacubitril-valsartan. There were no signs or symptoms of infection, adrenal insufficiency, liver failure or decompensated heart failure, and his vital signs, including heart rate and blood pressure, were normal. His serum glucose was $3.3 \mathrm{mmol} / \mathrm{L}$ and this presentation was attributed to decreased oral intake. After treatment with intravenous dextrose (50\% in water), his glucose increased to $13 \mathrm{mmol} / \mathrm{L}$. Over the course of an hour, his symptoms resolved with no recurrence of hypoglycemia and he was discharged.

Three weeks later, the patient presented to the emergency department with a spontaneous seizure and was found to have a serum glucose of $1.6 \mathrm{mmol} / \mathrm{L}$. He had no other acute symptoms. After treatment with intramuscular glucagon for rapid correction of his hypoglycemia, he was admitted to internal medicine.

The patient had also been feeling weak for the previous month, which he attributed to heart failure. At home, he had been eating usual portions of his traditional Lebanese diet. He normally ate lunch at $14: 00$ and both presentations to hospital occurred around 17:00. He did not have any early morning symptoms. He had no history of mental illness or access to antihyperglycemic medications (e.g., insulin or sulfonylureas) and did not consume alcohol. He had no previous surgeries, including gastric surgery.

Initial investigations, including blood count, blood cultures, electrolytes, liver enzymes and morning cortisol, were normal. The patient's renal function was at his baseline with an estimated glomerular filtration rate of $40 \mathrm{~mL} / \mathrm{min}$. Of note, his hemoglobin $A_{1 c}$ was 5.5\%, which had decreased from $6.2 \%$ the year before at an annual screening visit. Results from a urine sulfonylurea screen were negative for acetohexamide, chlorpropamide, tolazamide, tolbutamide, glimepiride, glipizide, glyburide, nateglinide and repaglinide. As the patient had presented with a first seizure, we performed computed tomography $(C T)$ of his head, which was normal.

\section{Key points}

- The approach to hypoglycemia in patients without diabetes differs depending on whether the patient is unwell and requires admission to hospital, or well and can be managed in the community.

- Assess the patient for the presence of Whipple triad.

- Ask about diet, medications and symptoms of neuroglycopenia, including generalized fatigue, visual disturbances, confusion, seizures and autonomic dysfunction.

- To aid diagnosis during a hypoglycemic episode, first-line investigations include laboratory investigations for levels of serum glucose, $C$-peptide, insulin and $\beta$-hydroxybutyrate; also consider screening for sulfonylurea and meglitinide and insulin antibodies.

- Although surreptitious medication effects should be considered, insulinoma is the most serious diagnosis to exclude in patients who appear otherwise well.

At this admission to hospital, the patient continued to have hypoglycemia over the course of the day, which necessitated continuous infusions of intravenous dextrose. Further investigations found an elevated serum insulin of 187 (normal range 35-140) $\mathrm{pmol} / \mathrm{L}$ and C-peptide of 8.18 (normal range 0.30 1.32) $\mathrm{nmol} / \mathrm{L}$, despite a low serum glucose of 2.1 (normal 3.311.0) $\mathrm{mmol} / \mathrm{L}$. Given that he appeared well, and with history and initial investigations not finding common causes of hypoglycemia such as sepsis, alcohol or medications, our working diagnosis was endogenous hyperinsulinemia possibly secondary to an insulinoma.

A conventional, noncontrast CT scan of the patient's abdomen was unremarkable. However, given high suspicion of an insulinoma, he underwent an indium-111 pentetreotide singlephoton emission computed tomography (SPECT)-CT scan that showed a $5.9 \mathrm{~cm} \times 4.1 \mathrm{~cm}$ intensely octreotide-avid mass near the pancreatic tail, and a small octreotide-avid hepatic mass (Figure 1). These findings were consistent with a large pancreatic insulinoma with metastatic liver deposit.

We deemed the patient to be a high-risk surgical candidate because of his cardiopulmonary comorbidities and we initially managed his condition with diazoxide, a nondiuretic benzothiadiazine. 

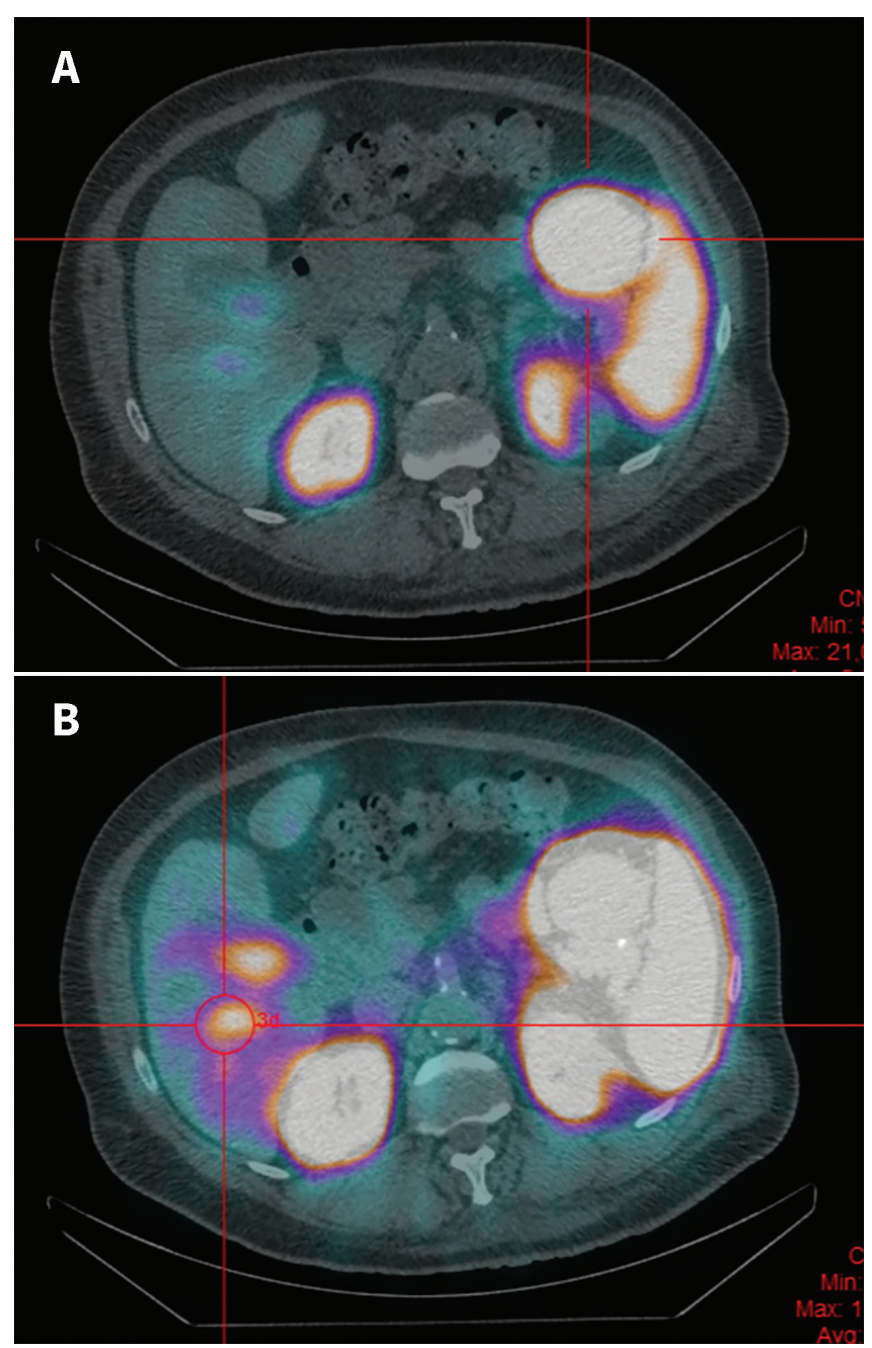

Figure 1: Indium-111 pentetreotide single-photon emission computed tomography-computed tomography (SPECT-CT) scan showing (A) an abnormal large octreotide-avid mass near the tail of pancreas (red circle) in a 67-year-old man who presented to the emergency department with a 2-hour history of generalized weakness, nausea and confusion and (B) a small hepatic deposit with octreotide activity suggestive of metastasis (red circle). Other bright masses are the normal appearance of kidneys and spleen on SPECT-CT.

Despite higher-level dosing of diazoxide, he continued to require intravenous dextrose infusions. We then switched to short-acting subcutaneous octreotide, a potent synthetic somatostatin analogue. With a standard starting dose of $100 \mu \mathrm{g}$, immediate resolution of hypoglycemia was achieved, and the dosage was titrated to $6 \mu \mathrm{g}$ twice daily to achieve normoglycemia. Under outpatient follow-up with neuroendocrine oncology, the patient was switched to long-acting intramuscular octreotide at a dosage of $20 \mathrm{mg}$ monthly, which continues to be effective in preventing hypoglycemia. Owing to renal insufficiency, he was not eligible for radionuclide therapy with ${ }^{177}$ Lu-DOTA-TATE but did receive external beam radiation to the mass near his pancreatic tail. We continue to monitor the mass with positron emission tomography-CT every 6 months, and its size has decreased following radiation.

\section{Discussion}

Hypoglycemia is a common presentation in the primary care setting and accounts for about 16 of every 1000 visits to the emergency department. ${ }^{1}$ In patients without diabetes, it is important to rule out common precipitating factors such as medications (e.g., indomethacin or antibiotics), alcohol, caloric restriction and systemic illness. In the absence of an obvious cause, further investigation for causes of endogenous hyperinsulinemia is warranted.

Although uncommon with an incidence of 4 per 1 million personyears, insulinomas are the most common functioning pancreatic neuroendocrine tumour and the most common cause of endogenous hyperinsulinemia. ${ }^{2}$ Most insulinomas are benign, well-differentiated tumours; malignant disease occurs in $5 \%-10 \%$ of cases. ${ }^{3}$ Patients usually present with fasting hypoglycemia, which may manifest with neuroglycopenic symptoms such as generalized fatigue, visual disturbances, confusion and seizures or autonomic symptoms including tremors, palpitations and diaphoresis. Our patient never had fasting hypoglycemia; both episodes occurred within hours after lunch. His second presentation with seizure and markedly low serum glucose suggested underlying pathology, prompting further workup for endogenous hyperinsulinemia.

\section{Approach to the patient with hypoglycemia}

Hypoglycemia, defined by Whipple triad, includes a serum glucose level of less than $3.1 \mathrm{mmol} / \mathrm{L}$, symptoms or signs consistent with hypoglycemia and resolution of the symptoms with correction of the hypoglycemia. ${ }^{4}$

One approach to determining the cause of hypoglycemia involves dividing patients into 2 groups: those who are systemically unwell and those who appear well (Table 1$).{ }^{4}$ The differential diagnosis in unwell patients includes organ failure (i.e., hepatic, cardiac or renal), sepsis, adrenal insufficiency, prolonged malnutrition and non-islet cell tumours producing insulin-like growth factor II. The differential diagnosis for patients who appear well includes insulinoma, insulin autoimmunity, noninsulinoma pancreatogenous hypoglycemia syndrome and hypoglycemia after gastric bypass. Clinicians should ask if the patient has taken medications known to cause hypoglycemia such as antihyperglycemic agents (e.g., sulfonylureas or meglitinides), exogenous insulin and other non-diabetic medications. More than 160 different medications have been associated with hypoglycemia, but the most common offending agents are quinolone antibiotics, pentamidine and quinine. ${ }^{5}$ Excessive alcohol use leading to hypoglycemia in a patient who does not use insulin is uncommon and a diagnosis of exclusion. Hypoglycemia after gastric bypass is characterized by episodic postprandial hypoglycemia. This occurs because of dumping syndrome, which is caused by rapid gastric emptying that leads to oversecretion of pancreatic hormones. Hypoglycemia after gastric bypass is primarily managed with dietary modifications, and pharmacotherapy is reserved for those with refractory disease. ${ }^{4}$ 
Table 1: Differential diagnosis for hypoglycemia in the well and unwell patient ${ }^{4 *}$

\section{Appears well}

1. Endogenous hyperinsulinemia

-Insulinoma

-Insulin secretagogue

-Non-insulinoma pancreatogenous hypoglycemia

-Post-gastric bypass hypoglycemia

-Insulin autoimmune hypoglycemia

2. Accidental, surreptitious or malicious hypoglycemia

\section{Appears unwell}

1. Medications/drugs

-Exogenous insulin

-Insulin secretagogues (sulfonylureas, meglitinides)

-Alcohol

-Other (indomethacin, pentamidine, quinine, lithium)

2. Critical illness

-Sepsis

-Cardiac, renal or hepatic failure

3. Hormone deficiency

-Adrenal insufficiency

-Glucagon deficiency

-Growth hormone deficiency

4. Non-islet cell tumour

*Reprinted from European Journal of Medicine, Vol. 25(5), Pieter Martens, Jos Tits, Approach to the patient with spontaneous hypoglycemia, p. 415-421, Copyright (2014), with permission from Elsevier.

\section{Hypoglycemia workup}

After confirming Whipple triad, laboratory investigations for the cause of hypoglycemia, including endogenous insulin overproduction, should be ordered. Initial investigations at the time of a hypoglycemic episode include levels of serum glucose, C-peptide, insulin and $\beta$-hydroxybutyrate. A sulfonylurea and meglitinide screen and insulin antibodies should also be ordered for patients without an identifiable cause of hypoglycemia. ${ }^{4}$ The results for the medication screen and insulin antibodies were negative in this patient.

Insulin is generated in the pancreas when its precursor, proinsulin, is cleaved into insulin and C-peptide. $\beta$-Hydroxybutyrate is a measurable ketone produced in the liver by the breakdown of fat in an insulin-deficient state when the supply of glucose is too low for metabolic processes, such as during periods of prolonged exercise, starvation or a carbohydrate-low diet. In patients with insulin resistance, insulin and C-peptide may be elevated without low serum glucose; therefore, insulin and C-peptide levels are uninterpretable without a corresponding glucose level. In patients with insulinoma, endogenous insulin overproduction occurs independent of serum glucose level, resulting in elevated insulin and C-peptide levels, which precipitates hypoglycemia (Table 2). Conversely, patients with exogenous hyperinsulinemia have high serum insulin levels with low C-peptide levels because pancreatic insulin production is inhibited. ${ }^{6} \beta$-Hydroxybutyrate is suppressed in both instances because of the antiketogenic effects of insulin, which can differentiate insulin-mediated from non-insulin-mediated hypoglycemia. As many laboratories do not detect insulin analogues, discussion with the local biochemistry department regarding available assays is suggested if exogenous insulin use is suspected.

A 72-hour fast may be required to reproduce hypoglycemia in a patient who is not symptomatic at the time of examination. This involves a protocolized supervised fast with serial measurements of glucose, insulin, C-peptide and $\beta$-hydroxybutyrate at regular intervals until the patient has documented hypoglycemia. This test is $99 \%$ sensitive for insulinoma. ${ }^{7}$

Table 2: Interpretation of laboratory results during an episode of hypoglycemia (serum glucose $<3.0 \mathrm{mmol} / \mathrm{L}$ ) ${ }^{4 \star}$

\begin{tabular}{|c|c|c|c|c|c|}
\hline Diagnosis & $\begin{array}{l}\text { Insulin } \\
\text { (pmol/L) }\end{array}$ & $\begin{array}{l}\text { C-peptide } \\
\text { (nmol/L) }\end{array}$ & $\begin{array}{c}\beta \text {-OH butyrate } \\
\text { (mmol/L) }\end{array}$ & $\begin{array}{l}\text { Insulin } \\
\text { antibody }\end{array}$ & $\begin{array}{c}\text { Circulating } \\
\text { oral antihyperglycemic }\end{array}$ \\
\hline Normal & $<20.8$ & $<0.2$ & $>2.7$ & Negative & No \\
\hline Exogenous insulin & $>>20.8$ & $<0.2$ & $<2.7$ & $\begin{array}{l}\text { Positive or } \\
\text { negative }\end{array}$ & No \\
\hline Insulinoma, NIPHS, PGBH & $\geq 20.8$ & $\geq 0.2$ & $<2.7$ & Negative & No \\
\hline Sulfonylurea overdose & $>>20.8$ & $\geq 0.2$ & $<2.7$ & Negative & Yes \\
\hline Insulin autoimmune & >> 20.8 & $>0.2$ & $<2.7$ & Positive & No \\
\hline IGF mediated & $<20.8$ & $<0.2$ & $<2.7$ & Negative & No \\
\hline Non-insulin mediated & $<20.8$ & $<0.2$ & $>2.7$ & Negative & No \\
\hline
\end{tabular}




\section{Localization of suspected insulinoma}

Noninvasive imaging modalities for localization include abdominal ultrasonography, conventional CT and magnetic resonance imaging (MRI), with MRI historically being the most sensitive of the conventional modalities. ${ }^{6}$ Nuclear medicine studies with somatostatinlabelled radiotracers, such as octreotide scintigraphy, have been effective in localizing neuroendocrine tumours. ${ }^{68} \mathrm{Ga}$-Exendin-4 PETCT is $84.6 \%$ sensitive for insulinomas when used alone, and $92.3 \%$ sensitive in combination with MRI, but has limited availability in North America. ${ }^{8}$ Invasive approaches such as endoscopic ultrasonography or hepatic vein sampling are generally reserved until after noninvasive measures have been explored. ${ }^{9}$ As we did not use contrast dye in this patient because of his impaired renal function, the initial CT did not detect the lesions. Thus, we proceeded with an octreotide scan that detected the pancreatic and hepatic masses.

Tissue biopsy is typically performed for confirmation of suspected insulinoma. However, we did not pursue biopsy for our patient because it would have required endoscopic ultrasonography and his medical comorbidities placed him at a high risk of complications. We made the diagnosis on the biochemical evidence of endogenous insulin overproduction, absence of other hormone overproduction and a positive result for the functional octreotide scan.

\section{Management of insulinoma}

The definitive treatment of insulinoma is surgical resection. Most insulinomas are benign and confer an excellent prognosis after tumour resection, with disease-specific survival of $98 \%$ at 1 year, $92 \%$ at 5 years and $90 \%$ at 10 years. However, patients with unresectable malignant disease have a much poorer longterm prognosis, with disease-specific survival of $82 \%$ at 1 year and $25 \%$ at 5 and 10 years. ${ }^{10}$

When surgery is not possible, the goal of treatment is to mitigate hypoglycemia with dietary modification, pharmacotherapy (e.g., diazoxide, somatostatin analog or everolimus) or minimally invasive cytoreductive therapies. Diazoxide is first-line pharmacotherapy; somatostatin analogs such as octreotide are reserved for when diazoxide is ineffective at maintaining euglycemia. Longacting octreotide formulations are used for maintenance therapy, as in our patient. ${ }^{11}$

Everolimus is a mammalian target of rapamycin (mTOR) inhibitor that has been used infrequently for the treatment of metastatic pancreatic neuroendocrine tumours. Chemotherapy agents such as streptozocin and doxorubicin have also been reported to have varying effectiveness. ${ }^{11}$ However, few cases included in studies have been specific to insulinoma, and more data on the application of these agents are required. ${ }^{11}$

\section{References}

1. Kumar JG, Abhilash KPP, Saya RP, et al. A retrospective study on epidemiology of hypoglycemia in emergency department. Indian J Endocrinol Metab 2017; 21:119-24.

2. Metz DC, Jensen RT. Gastrointestinal neuroendocrine tumors: pancreatic endocrine tumors. Gastroenterology 2008;135:1469-92.

3. Giannis D, Moris D, Karachaliou GS, et al. Insulinomas: from diagnosis to treatment. A review of the literature. J BUON 2020;25:1302-14.
4. Martens P, Tits J. Approach to the patient with spontaneous hypoglycemia. Eur J Intern Med 2014;25:415-21.

5. Murad MH, Coto-Yglesias F, Wang AT, et al. Drug-induced hypoglycemia: a systematic review. J Clin Endocrinol Metab 2009;94:741-5.

6. Abboud B, Boujaoude J. Occult sporadic insulinoma: localization and surgical strategy. World J Gastroenterol 2008;14:657-65.

7. Service FJ, Natt N. The prolonged fast. J Clin Endocrinol Metab 2000;85:3973-4.

8. Antwi K, Nicolas G, Fani M, et al. ${ }^{68} \mathrm{Ga}$-Exendin-4 PET/CT detects insulinomas in patients with endogenous hyperinsulinemic hypoglycemia in MEN-1. J Clin Endocrinol Metab 2019;104:5843-52.

9. Okabayashi T, Shima Y, Sumiyoshi T, et al. Diagnosis and management of insulinoma. World J Gastroenterol 2013;19:829-37.

10. Sada A, Glasgow AE, Vella A, et al. Malignant insulinoma: a rare form of neuroendocrine tumor. World J Surg 2020;44:2288-94.

11. Matej A, Bujwid H, Wroński J. Glycemic control in patients with insulinoma. Hormones (Athens) 2016;15:489-99.

Competing interests: Mahua Ghosh has received consultant fees from the Diabetes Canada Program Planning Committee and the Saskatchewan Cancer Agency, and support for travel or attending meetings from the University of Alberta (academic funding) and the Alberta Medical Association. She is a Council of the Canadian Society of Endocrinology and Metabolism and a member of the Specialty Committee for Endocrinology and Metabolism of the Royal College of Physicians and Surgeons of Canada. She is a member of PCOS Together (a public forum for polycystic ovarian syndrome). No other competing interests were declared.

This article has been peer reviewed.

The authors have obtained patient consent.

Affiliations: Division of Endocrinology and Metabolism (Vu, Mereu, Ghosh) and Division of Internal Medicine (Wang), Department of Medicine, University of Alberta, Edmonton, Alta.

Contributors: Tianqi Wang and Albert Vu prepared the initial manuscript. Mahua Ghosh was involved in the chart review. All of the authors contributed to the design of the work, critically revised the manuscript for important intellectual content, gave final approval of the version to be published and agreed to be accountable for all aspects of the work.

Content licence: This is an Open Access article distributed in accordance with the terms of the Creative Commons Attribution (CC BY-NCND 4.0) licence, which permits use, distribution and reproduction in any medium, provided that the original publication is properly cited, the use is noncommercial (i.e., research or educational use), and no modifications or adaptations are made. See: https://creativecommons.org/ licenses/by-nc-nd/4.0/

Funding: Mahua Ghosh has received a grant from the Women and Children's Health Research Institute (paid to her institution).

Correspondence to: Tianqi Wang, tianqi5@ualberta.ca

The section Cases presents brief case reports that convey clear, practical lessons. Preference is given to common presentations of important rare conditions, and important unusual presentations of common problems. Articles start with a case presentation (500 words maximum), and a discussion of the underlying condition follows (1000 words maximum). Visual elements (e.g., tables of the differential diagnosis, clinical features or diagnostic approach) are encouraged. Consent from patients for publication of their story is a necessity. See information for authors at www.cmaj.ca. 